\title{
Wireless Positioning Algorithm Based on RSS in Limited Space
}

\author{
Yongxing $\mathrm{Wang}^{1}$, Gang Hua ${ }^{1,2, *}$ Yonggang $\mathrm{Xu}^{3,4}$ and Hongsheng $\mathrm{Yin}^{3,5}$ \\ ${ }^{1}$ China University of Mining and Technology \\ ${ }^{3}$ China University of Mining and Technology \\ 'wyx_783@163.com, ${ }^{2}$ ghua3323@163.com, feilongxyg@163.com, \\ 5xuzhouyhs@sina.com
}

\begin{abstract}
Positioning algorithm RSS-based (Received Signal Strength) has become a research hotspot of wireless location technology because it decreases hardware costs, consumes less energy, and implements simplicity etc. However, wireless signal can cause data fluctuation of RSS easily because of shadowing and multipath in the actual environment, especially in the limited space such as underworkings whose characteristics are humid, dusty, and severe shadow. So the wireless signal is easier to produce the multipath fading for refraction, reflection, and scattering. The new challenge about RSS-based positioning algorithm are brought by these factors in the limited space. In this study, a novel RSS-based positioning algorithm is proposed. Firstly, during the RSS collected process, the RSS fingerprint database is established based on supervised methods to ensure its precision; Secondly, during the positioning process, a variance filter is designed for correcting the target's RSS to ensure reliability of the RSS. Experiment results show that the feasibility and effectiveness of proposed algorithm are verified, and thereby the ideal accuracy.
\end{abstract}

Keywords: Wireless Localization; Signal Strength; Fingerprint Information; Limited space

\section{Introduction}

As the Wireless Sensor Networks (WSN) applications are increasingly widespread, they have been applied to many areas in the production and life: such as ecological environment monitoring, anti-terrorism information collection, fire and earthquake rescue personnel, the information acquisition of the coal mine, etc.[1,2], which have deployed a large number of wireless sensors in. If the collected data don't includes coordinate information, they aren't significant data. Therefore, wireless positioning technology based on Wireless Local Area Networks (WLAN) has become an important research part of mobile internet in recent years. The RSS-based positioning algorithms which require single parameter in the localization become a research hotspot. A lot of researched works have been done at home and aboard, and they can be divided into two categories: positioning algorithms based on the probabilistic model [3] and the deterministic model [4].

The main idea of probability positioning model constructs a conditional probability distribution between signal strength and location in the application environment. In [5], probability inference methods were adopted for the mote positioning, and the signal intensity probability distribution of each location was calculated by the RSS information in the positioning region. The fluctuation of the user location was effectively limited

* Corresponding Author 
according to the introduction of the space constraints of the user motion trajectory. In the work of [6], Hidden Markov Model (HMM) was applied to target trajectory model, and adopted state transition matrix to express transfer relation among the location, and then the most max probability trajectory sequence was acquired by using Viterbi algorithm. In[7], the probability of particle filter method was applied to positioning, not only did the localization accuracy can be acquired the same as deterministic model, but also the multiple sensor information was effectively fused together. In the work of [8] the nearest neighbor method was compared with Naive Bayes method and histogram distribution probability, respectively, experimental results showed that the positioning algorithm based on probability ware better than nearest neighbor algorithm.

Deterministic localization model is that the mote location was solved out by using certainty inference method. Channel State Information (CSI) was firstly proposed as a new fingerprint parameters in the positioning area[9]. CSI not only provided more multi-dimensional information, but localization accuracy was also more stable performance and positioning results than the signal strength parameters. $\operatorname{In}[10]$, decision tree was introduced to decisive the mote localization. Firstly, all of the users were clustered by the environment in the algorithm they belong to, and then the decision tree was constructed in each category according to the size of information entropy about Access Point (AP). During the online positioning process, firstly the target was determined to which class it belonged to, and then fine-grained target location was solve by using the decision tree in the category. Support vector machine (SVM) was adopted for a means of learning [11], and fused the computer vision technology to help the target' positioning, and got a better location performance. In the work of [12] used fine-grained CSI to realize high precision positioning with the WIFI signal wavelength level, and the motes consumed lower energy in the algorithm than traditional positioning method. $\operatorname{In}[13]$, $k \square$ nearest neighbor algorithm was used to estimate the mote location, the method was that signal intensity vector would be collected in real time, it matched with the signal intensity vector in the all of the training data set, and it finally selected the location coordinates for most similar RSS whose coordinates as the mote estimation coordinate.

Relative application research in wireless positioning is less in the field of coal mine than others. the main reason lies in the coal mine environment which belongs to the limited space $[14,15]$, coal mine includes humid air, coal dust, and object shielding et al., the environment makes the wireless signal be easy to cause reflection, refraction, scattering which causes multipath effect making larger RSS fluctuation, so implemented the wireless positioning in the coal mine environment has brought new technology challenges. In [16], the underworkings space was divided into many gateway-centric distributed local positioning units, the coordinates in the local positioning units ware estimated by using RSS-based distance measurement method, and three-dimensional coordinates of the underworkings network were acquired through coordinates transformation; therefore, the information (e.g., speed, direction, and location etc.) can be confirmed in the monitoring units. Finally, the coordinates of blind area about the motes can be estimated by estimating algorithm. In [17], a weighted centroid localization algorithm was put forward based on RSS information. First of all, the path decline index was dynamically accessed by calculating path decline index in the located area, it responded precisely influence about signal loss in the different positioning area, and enhanced the adaptive capacity of the environment for distance measurement algorithm, the coordinate was calculated by weighted centroid algorithm at last.

However, underworkings is relatively simpler than other environment. There is no other target except miners and locomotive. This paper combines with the characteristics of the coal mine underworkings, a new localization algorithm based on location fingerprint information is proposed. The experimental results show that proposed algorithm solved existing problems (e.g., low accuracy, bad real-time) effectively in the coal mine 
environment.

\section{Established the Location Fingerprint Database}

\subsection{Collected Data Algorithm about the RSS Information}

Wireless Access Point (AP) is also called beacon nodes (or anchor nodes) which cover the positioning area, which are divided into many located reference points (LPP) $i(i=1,2, \mathrm{~L}, n)$, RSS of the $i^{\text {th }} \mathrm{LPP}$ is received from the AP as the $R_{i}$, and RSS of the $i^{\text {th }}$ LPP is received from the $J^{\text {th }}(J=1,2, \cdots)$ AP as the $R_{A J_{i}} \cdot R_{A J_{i}}$ is called as one component of $R_{i}$, using the vector to express as the $R_{i}\left(R_{A 1_{i}}, R_{A 2_{2}}, \mathrm{~L}, R_{A J_{i}}\right)$.

Collecting $M$ times data $R_{i}$ on LPP may conduct $m(m \leq M)$ times of them caused distortion because wireless signal is influenced by multipath and shadowing (distortion: It may be one or more of these components caused distortion, whose value may be is larger or smaller than the its true value over some range), so collected $m$ times data couldn't be the gist for generating location fingerprint information. In view of this, a mean filter is designed to remove these distorting values, described as follows:

(1) The average values of the $M$ times are calculated on the $i^{\text {th }}$ LPP, as:

$\overline{R_{A J_{i}}}=\sum_{j=1}^{M} R_{A J_{i_{j}}} \quad i=1,2, \cdots, n ; J=1,2, \cdots$

(2) The distorting coefficient of collected the $j^{\text {th }}$ data is $\chi_{i_{j}}(j=1,2, \cdots, \mathrm{M})$ on the $i^{\text {th }}$ LPP, $\chi_{i_{j}}$ is given as follows:

$\chi_{i_{j}}=\sqrt{\left(R_{A 1_{i_{j}}}-\overline{R_{A 1_{i}}}\right)^{2}+\left(R_{A 2_{i_{j}}}-\overline{R_{A 2_{i}}}\right)^{2}+\cdots+\left(R_{A J_{i_{j}}}-\overline{R_{A J_{i}}}\right)^{2}}$

(3) Remove the $m$ times distorting data on the $i^{\text {th }}$ LPP: set a threshold $\gamma$, whose value is decided by actual communication environment and positioning accuracy requirement.

$\left\{\begin{array}{c}\chi_{i j} \geq \gamma \quad \text { discard } R_{A J_{i j}} \\ \chi_{i j}<\gamma \quad \text { retain } R_{A J_{i j}}\end{array}\right.$

(4) By analogy from collected $M$ times data, the last $M-m$ times data as a gist to establish RSS fingerprint.

\subsection{Constructed Location Fingerprint Algorithm}

The rest of $M-m$ times data on the $i^{\text {th }}$ LPP can represent RF signal strength of this point (i.e., spatial distribution of the RF signal at this point). However, as far as statistics theory knowledge is concerned, the common characteristic can be more precisely expressed by the group average probability characteristic. So the average of the $M-m$ times collected data is the location fingerprint at the $i^{\text {th }}$ LPP. Set location fingerprint at the $i^{\text {th }}$ point is $R_{F_{i}}, R_{F_{i}}$ can be given as follows: 
$R_{F_{i}}=\frac{1}{M-m} \stackrel{\mathrm{a}}{M-m}_{k=1}^{\stackrel{\circ}{\circ}} R_{A_{i_{k}}}$

where $i=1,2, \cdots$ and $M$ is a natural number greater than zero. $R_{F_{i}}$ is expressed by vector as $R_{F_{i}}\left(R_{F_{-} A 1_{i}}, R_{F_{-} A 2_{i}}, \mathrm{~L}, R_{F_{-} A J_{i}}\right)$, which is called as fingerprint data. So the above formula (4) can be changed as:

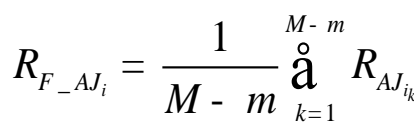

Therefore, the formula (4) combines with formula(5), a new modality is generated as follows:

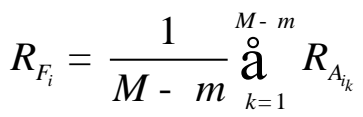

$$
\begin{aligned}
& =\frac{1}{M-m} \stackrel{\mathrm{a}}{\mathrm{a}=1}^{M-m}\left(R_{A 1_{i_{k}}}, R_{A 2_{i_{k}}}, \mathrm{~L}, R_{A{J_{i}}_{i_{k}}}\right) \\
& =\left(\overline{R_{A 1_{i}}}, \overline{R_{A 2_{i}}}, \mathrm{~L}, \overline{R_{A J_{i}}}\right) \\
& =\left(R_{A 1_{i}}, R_{A 2_{i}}, \mathrm{~L}, R_{A J_{i}}\right)
\end{aligned}
$$

The location fingerprint data at all the LPP could be generated by taking turn $i=1,2, \cdots$, so the location fingerprint database can be produced by them, and location fingerprint vector is expressed as the $\left[\left(x_{i}, y_{i}, z_{i}\right), R_{F_{-} A 1_{i}}, R_{F_{-} A 2_{i}}, \cdots, R_{F_{-} A J_{i}}\right]$ in the location fingerprint database.

The algorithm flow for constructing fingerprint database is shown in Figure 1

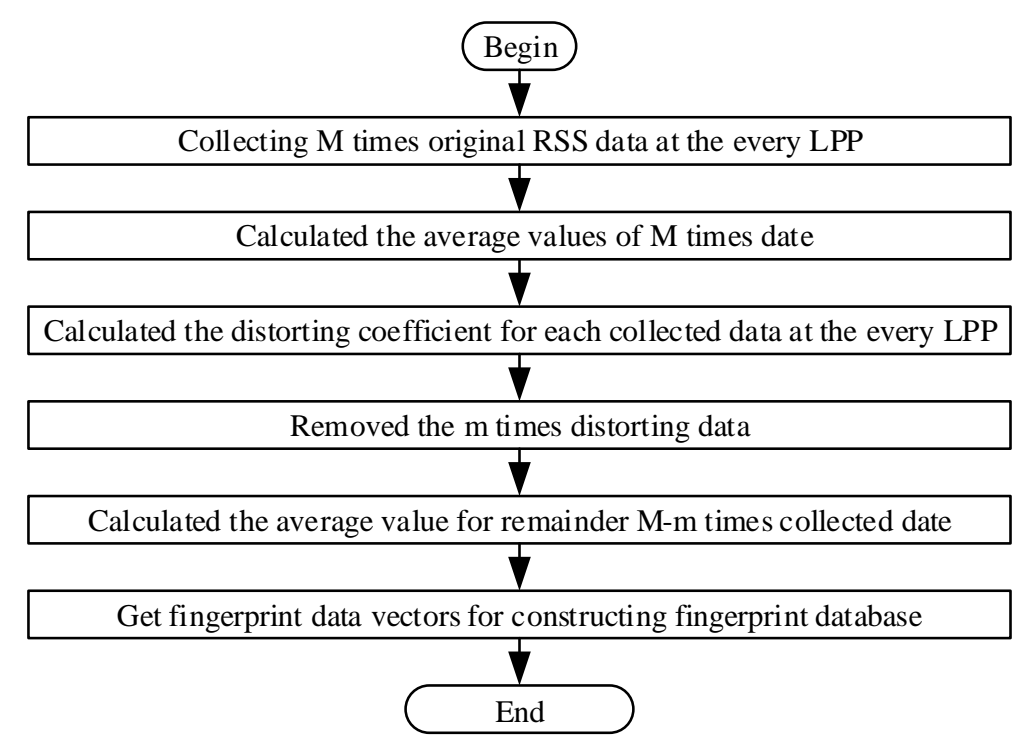

Figure 1. The Process Flow for Constructing Fingerprint Database 


\section{Positioning Algorithm Based on Location Fingerprint}

Common nodes (i.e., non-anchor node without the location) receive currently RF signal strength about the each AP in the located area, these nodes send the RSS using their transmitter module afterwards to the principal computer, described the algorithm as follows:

1) Assuming that common nodes send data as $R_{L_{-} A J}(J=1,2, \mathrm{~L})$, which is

expressed by vectors $\left(R_{L_{-} \mathrm{A} 1}, R_{L_{-} \mathrm{A} 2}, \ldots, R_{L_{-} \mathrm{A} J}\right)$ called pending data. The $R_{F}$ expresses fingerprint database which is expressed by the vector $\left[\left(x_{i}, y_{i}, z_{i}\right), R_{F_{-} A 1_{i}}, R_{F_{-} A z_{i}}, \cdots, R_{F_{-} A J_{i}}\right]$.

2) Repairing the pending data: According to the previous discussion, location fingerprint data have been filtered once time during the generating stage. So the pending data absolutely possibly make distortion as well, if the pending data which have generated aren't disposed, it goes without saying that the positioning error will be very big, then the estimated coordinates are useless. Therefore, before estimating the local coordinate, pending data have been judged whether they have generated distortion.

Its main idea is that location fingerprint data $R_{F}$ is generated by a lot of $R_{A J_{j}}(J=1,2, \mathrm{~L} ; j=1,2, \mathrm{~L}, M-m)$ after filtering some distorting data. Therefore, $R_{F}$ are calculated after the average of these data is solved after removing the distorting data, so these pending data $R_{L_{-} \mathrm{A} J}(J=1,2, \mathrm{~L})$ should satisfy following the formula in theory:

$$
\begin{gathered}
R_{F_{-} A J_{\min }}=\min \left(R_{F_{-} A J_{i}}\right) \\
R_{F_{-} A J_{\max }}=\max \left(R_{F_{-} A J_{i}}\right) \\
\frac{1}{3} R_{F_{-} A J_{\min }}-x £ R_{L_{-} A J} £ R_{F_{-} A J_{\max }}+x
\end{gathered}
$$

Where $x$ is called the tolerability (the value of $x$ is derived from actual located environment and positioning accuracy). When the $R_{L_{-} \mathrm{AJ}}(J=1,2, \mathrm{~L})$ does not satisfy the above formula(7), the pending data are called the generating distort, then they are discarded and wait for a new pending data once again; if not, the next step is turn into.

3) Calculating the distance $\Delta d$ between pending data vector and location fingerprint vector, just as:

$$
\Delta d=\sqrt{\left(R_{L_{-} A}-R_{F_{-} A}\right)^{2}}
$$

Calculate further the above formula(8):

$$
\Delta d_{i}=\sqrt{\left(R_{L_{-} A 1}-R_{F_{-} A 1_{i}}\right)^{2}+\left(R_{L_{-} A 2}-R_{F_{-} A 2_{i}}\right)^{2}+\cdots+\left(R_{L_{-} A J}-R_{F_{-} A J_{i}}\right)^{2}}
$$

Where $\mathrm{D} d_{1}$ is calculated when $i=1$. So the rest of $\mathrm{D} d_{2}, \mathrm{D} d_{3}, \ldots, \mathrm{D} d_{i}$ can be calculated by changing all of the LPP $i$, solved minimum in the set $\mathrm{D} d$ :

$$
\Delta d_{\min }=\min \left(\Delta d_{1}, \Delta d_{2}, \cdots, \Delta d_{n}\right)
$$


As follows,

$\Delta d_{\min }=\Delta d_{k}$

Then, the estimating user coordinates is $\left(x_{k}, y_{k}, z_{k}\right)$.

The algorithm flow is as shown in figure 2:

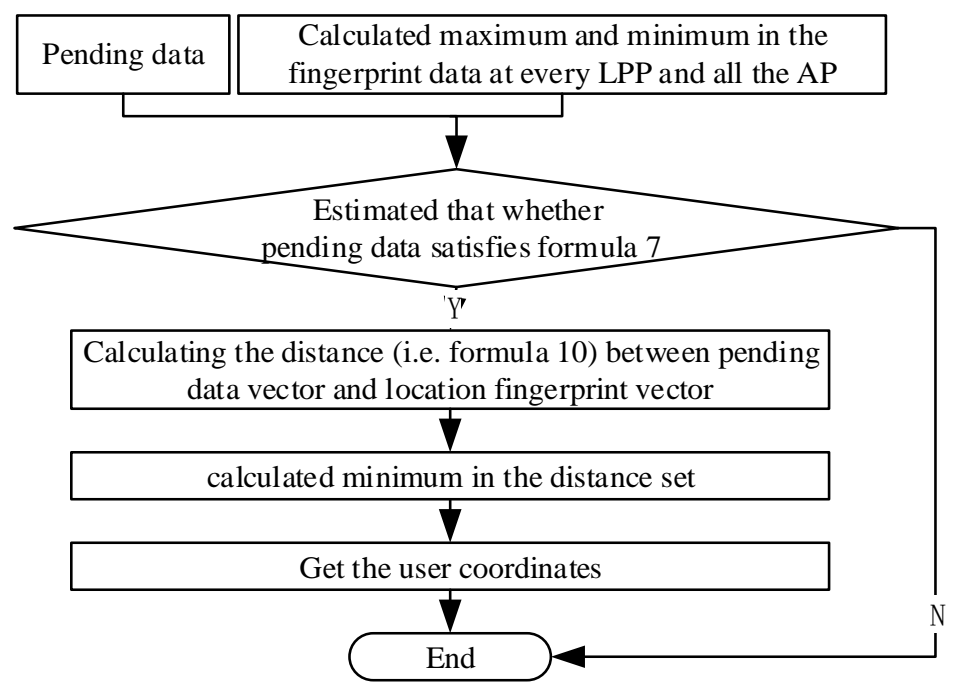

Figure 2. Process Flow for Positioning Algorithm

\section{The Experiment Simulation and Result Analysis}

\subsection{The Simulation Environment}

Experiment test was conducted in the air-raid shelter to evaluate the proposed algorithm performance. The air-raid shelter whose width, high and length are $2.5 \mathrm{~m}, 2.7 \mathrm{~m}$ and $100 \mathrm{~m}$, respectively. Its ground is covered by the cement, and the wall is coarse, two top sides are layout by some cable, the average temperature and relative humidity are respectively $24.5^{\circ} \mathrm{C}$ and $57.2 \%$ when the experiment is being done. So experiment environment is similar with actual underworkings about their characteristics. The beacon nodes $A P 1(7,1), A P 2(43,-1)$ and $A P 3(97,1)$ were deployed on the two sides of air-raid shelter. Its length was divided into an average of 100 LPP. Because underworkings' width is far less than its length, the coordinates can ignore its ordinate in the actual coal mine environment, so ordinate is also ignored when the coordinates are calculated in the experiment simulation.

As shown in Figure 3(a) that the type of common node is KJ226-K identification card. It is a kind of active label based on WIFI communication protocol, it can work for two years, and be appropriate for coal mine environment that has the gas and coal dust explosion gas. As shown in figure 3(b) that the type of beacon node is Ruckus ZoneFlex 7962 whose working frequency is $2.4 \mathrm{GHZ}$. It satisfies IEEE 802.1 standard and provides 54 MBPS data transmission rate, adopts orthogonal frequency division multiplexing technology and is specially designed for coal mine application, it has the characteristic of high speed and receiving far distance etc., in particular, it can be connected by different gain antenna to get different coverage. 


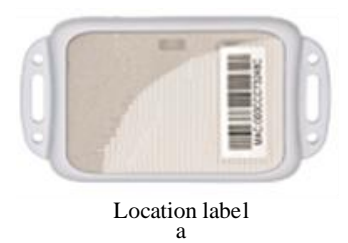

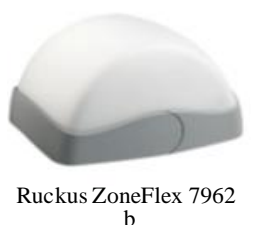

Figure 3. The Location Label and Anchor Node

\subsection{Experiment Results and Analysis}

4.2.1. Constructed location fingerprint information: As the distance increased, the RF signal strength decreases in the ideal space in Figure 4(a), Figure 4 (b), (c), (d) are corresponding RSS fingerprint data vector on the every LPP when $M-m$ is different values.
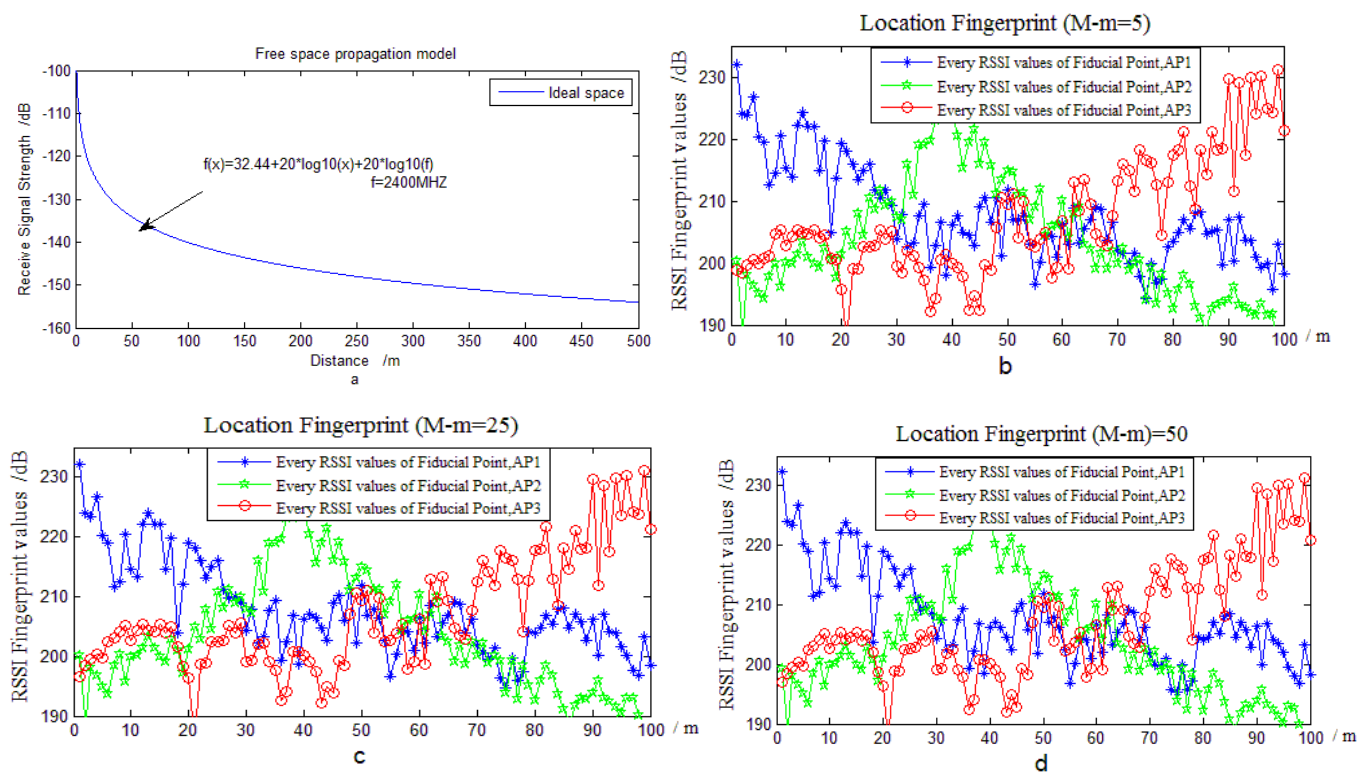

Figure 4. Location Fingerprint

4.2.2. Calculated positioning error: According to the fingerprint data vector constructed on the above section, user carries common node with a normal speed in the air-raid shelter. Estimated coordinates and actual coordinates are represented respectively by $\left(x^{\prime}, y^{\prime}\right)$ and $(x, y) . \operatorname{Err}_{i}$ and $\overline{E r} r_{i}$ represent respectively absolute estimated error and average error of 50 times. Err is ensemble average (a total 100 test points), their computed formulas are given respectively as follows:

$$
\begin{aligned}
& \operatorname{Err}_{i}=\sqrt{\left(x^{\prime}-x\right)^{2}+\left(y^{\prime}-y\right)^{2}} \\
& \overline{\operatorname{Err}_{i}}=\sum_{j=1}^{50} \operatorname{Err}_{i_{j}} \quad i=1,2, \cdots, 100
\end{aligned}
$$


$E r r=\frac{1}{100} \sum_{i=1}^{100} \overline{E r r}$

As shown in Figure 5 that the 50 times $\overline{E r r}_{i}$ of user node were derived respectively when $M-m=5, M-m=25, M-m=50$, and tolerability $\xi=1.2$, respectively.

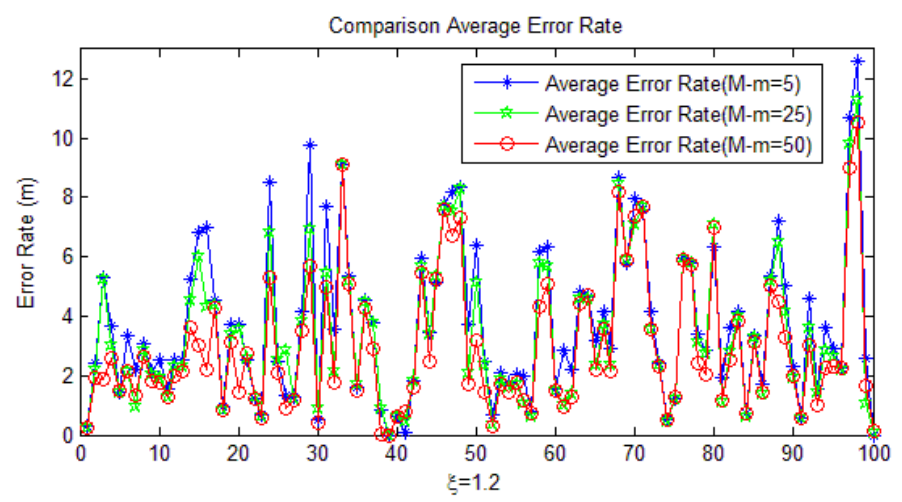

Figure 5. Experience Simulation Results

As shown in figure 5 that the $\overline{E r r_{i}}$ values decrease when the value of $\underline{M}-m$ increases at the overall situation. But the average error $\overline{E r r_{i}}$ on the $46^{\text {th }}, 47^{\text {th }}, 48^{\text {th }}, 68^{\text {th }}, 69^{\text {th }}, 70^{\text {th }}, 71^{\text {th }}, 97^{\text {th }}$ and $98^{\text {th }}$ LPP are larger than others; their data are severely undulate form the experiment data $R_{L_{-} A k}(k=1,2,3)$. These LPP are easy to take place in undulate areas through repeated testing. The main reason is that these localization areas have some shadowing, so they are liable to signal refraction and reflection. As shown in Table 1 that ensemble average error Err decreases along with $M-m$ values increasing, but the ensemble average error $E r r$ changes any more when $M-m$ value is in the range from 75 and 100 .

Table 1. Ensemble Average err Result of the System Simulation

\begin{tabular}{cc}
\hline M-m & ensemble average error Err $(\mathrm{m})$ \\
\hline $\mathbf{5}$ & 3.75 \\
$\mathbf{2 5}$ & 3.36 \\
$\mathbf{5 0}$ & 3.06 \\
$\mathbf{7 5}$ & 2.95 \\
$\mathbf{1 0 0}$ & 2.95 \\
$\mathbf{2 0 0}$ & 2.95 \\
\hline
\end{tabular}

However, in the larger positioning error area, the main cause of this result includes two aspects: one is positioning itself area environment (described above); the second is that the proposed positioning algorithm does not effectively process these distorting data when pending data have distorted. The latter is processed by the following two aspects, on the one hand, distorted data should be corrected effectively when RSS have generated distortion; on the other hand, the distorted data can be removed out when system can find them. A designed filter can effectively observe pending data whether they have generated distortion, so the parameter $x$ of filter is modified to test influence of the positioning accuracy. 
Table 2. Tolerability § Influences Ensemble Average Positioning Error

\begin{tabular}{ccc}
\hline \multirow{2}{*}{ M-m } & \multicolumn{2}{c}{ ensemble average positioning error Err $/ \mathbf{m}$} \\
\cline { 2 - 3 } & $\xi=1$ & $\xi=0.5$ \\
\hline 5 & $3.67 \mathrm{~m}$ & $3.5 \mathrm{~m}$ \\
25 & $3.30 \mathrm{~m}$ & $3.23 \mathrm{~m}$ \\
50 & $3.00 \mathrm{~m}$ & $3.02 \mathrm{~m}$ \\
75 & $2.93 \mathrm{~m}$ & 2.90 \\
100 & $2.93 \mathrm{~m}$ & 2.91 \\
200 & $2.94 \mathrm{~m}$ & 2.90 \\
\hline
\end{tabular}

As shown in Table 2, if the values of $M-m$ does not change, positioning accuracy is better when $x=0.5$ than that of $x=1$ in the localization area. If the values of $x$ do not change, positioning accuracy is better along with $M-m$ increasing. However, if the value of $M-m$ is greater than 50, the positioning accuracy does not have any change. That is to say, the number of sample comprised location fingerprint is larger than fixed value, positioning accuracy is not influenced by the number of sample.

The relation was studied between tolerability $x$ and ensemble average error Err. As is shown in Figure 6 that the value of ensemble average error Err decreases when tolerability $x$ is smaller. When the value of $x$ is much less in the range from $0 £ x £ 1$, the ensemble average error hardly changes (i.e., the positioning accuracy is high).

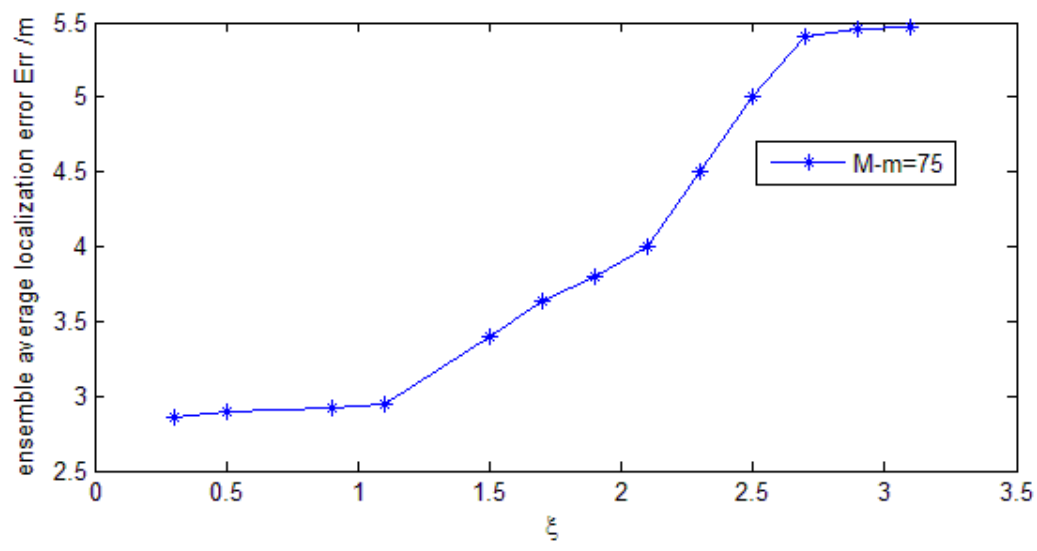

Figure 6. The Relation between the Three $\xi, t$, Err

4.2.3. Compared proposed algorithm performance with other algorithms of reference: The field trial was conducted to further verify the proposed localization algorithm performance in the same research background (coal mine). The proposed algorithm was compared with these algorithm in the literature [11] and [12] in some LPP. The parameters of the proposed algorithm are $M-m=75$ and $\xi=0.5$, the parameter area $n$ in [12] is derived dynamically. Experiment result were shown as Figure 7 and Table 3. 
Table 3. Performance Comparison of Positioning Algorithm

\begin{tabular}{|c|c|c|c|}
\hline Positioning Algorithm & $\begin{array}{c}\text { Real } \\
\text { Coordinate }\end{array}$ & Estimated Coordinate & Located Error \\
\hline Proposed Algorithm & & $(1,4)$ & 1.000 \\
\hline Located Algorithm in literature [11] & $(1,5)$ & $(2,7)$ & 2.236 \\
\hline (Area $\mathrm{n}+$ Weighted Centroid) in literature [12] & & $(1,6)$ & 1.000 \\
\hline Proposed Algorithm & & $(1,8)$ & 1.414 \\
\hline Located Algorithm in literature [11] & $(2,9)$ & $(2,7)$ & 2.000 \\
\hline (Area $\mathrm{n}+$ Weighted Centroid) in literature [12] & & $(3,7)$ & 2.236 \\
\hline Proposed Algorithm & & $(3,13)$ & 3.606 \\
\hline Located Algorithm in literature [11] & $(5,16)$ & $(5,10)$ & 6.000 \\
\hline (Area $\mathrm{n}+$ Weighted Centroid) in literature [12] & & $(6,11)$ & 5.100 \\
\hline Proposed Algorithm & & $(5,23)$ & 2.000 \\
\hline Located Algorithm in literature [11] & $(5,25)$ & $(7,28)$ & 3.606 \\
\hline (Area $\mathrm{n}+$ Weighted Centroid) in literature [12] & & $(6,26)$ & 1.414 \\
\hline Proposed Algorithm & & $(6,48)$ & 3.162 \\
\hline Located Algorithm in literature [11] & $(7,45)$ & $(5,41)$ & 4.472 \\
\hline (Area $\mathrm{n}+$ Weighted Centroid) in literature [12] & & $(4,46)$ & 3.162 \\
\hline Proposed Algorithm & & $(3,62)$ & 2.000 \\
\hline Located Algorithm in literature [11] & $(3,60)$ & $(4,57)$ & 3.162 \\
\hline (Area $\mathrm{n}+$ Weighted Centroid) in literature [12] & & $(3,58)$ & 2.000 \\
\hline Proposed Algorithm & & $(4,71)$ & 1.000 \\
\hline Located Algorithm in literature [11] & $(4,70)$ & $(3,69)$ & 1.414 \\
\hline (Area $\mathrm{n}+$ Weighted Centroid) in literature [12] & & $(4,73)$ & 3.000 \\
\hline
\end{tabular}

Simulated figure of located error was got as shown in Figure 7:

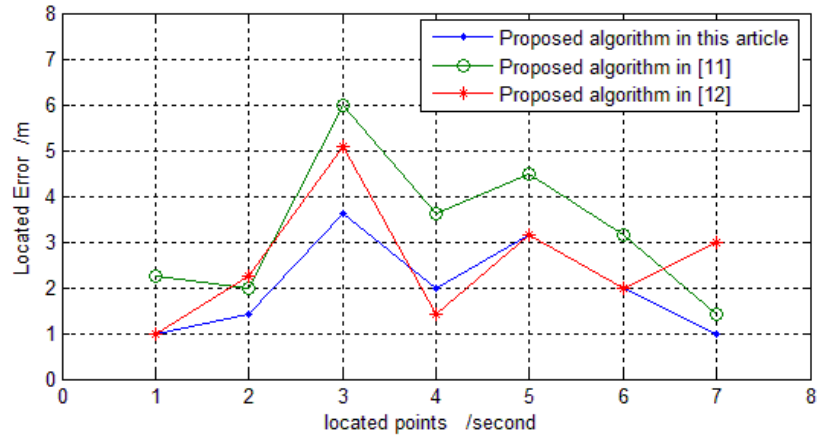

Figure 7. Located Error Comparison of Positioning Algorithm

Some conclusions can be drawn from the Table 3 and Figure 7 as follows:

1) In the same experiment environment, the estimated error of the proposed algorithm is better than that in literature [11] and [12]. As simulated result above, it is plausible to postulate that the proposed algorithm is better than previous algorithm.

2) The smaller the distance between user and AP is, the smaller the estimated error of positioning algorithm can be. The user close to AP receives the lager RSS value 
suffering from little multipath and shadowing effect. Therefore, if the condition is better, we can deploy more AP in a positioning system to improve localization accuracy to a certain degree.

\section{Conclusion}

1) Severe multipath and shadowing exist in the limited space such as coal mine, RSS is liable to distort. Therefore, a lot of sample data are collected to comprise location fingerprint database, but they should filter to ensure that the location fingerprint is precise.

2) Designed filter can effectively judge the pending data whether they have distorted during the positioning process. The user coordinates which are significant are derived from these pending data.

3) Experiment simulation results shown that the minimum ensemble average estimated error is $2.86 \mathrm{~m}$, and the parameter $x$ and $\mathrm{M}-\mathrm{m}$ are analyzed from the multiple perspective to analyze influence for positioning accuracy. The problems just as lower localization accuracy are solved perfectly.

4) Further study on how to dynamically intellectually adjust $x$ value on the every LPP to improve positioning accuracy will be started as soon as possible.

\section{Acknowledgment}

This work is partially supported by National Natural Science Foundation of China (No.61379100, No. 51204186) and the Fundamental Research Funds for the Central Universities (No. 2010QNB30)

\section{References}

[1] G. He, L. Li and L. Lao, "A Localization Algorithm Based on Geographical Similarity of RSSI in Wireless Sensor Networks", Computer Network and Multimedia Technology, Cnmt, International Symposium on. IEEE, Wuhan, China, (2009) December 18-18.

[2] H. Shi, "A new weighted centroid localization algorithm based on RSSI. Information and Automation (ICIA)”, 2012 International Conference on, Shenyang, China, (2012) January 06-08.

[3] X. Yi, Y. Liu and L. Deng, "A Novel Environment Self-adaptive Localization Algorithm Based on RSSI for Wireless Sensor Networks", 2010 IEEE International Conference on Wireless Communications Networking and Information Security (WCNIS), Beijing, China, (2010) January 25-27.

[4] XY. Ding, H. Zhao, J. Zhu, K. Zhang, DZ. Li, "A Novel Localization Algorithm Based on RSSI for Wireless Sensor Networks", 2011 7th International Conference on Wireless Communications Networking and Mobile Computing (WICOM), Wuhan, China, (2011) September 23-25.

[5] A M Ladd, K. E. Bekris, A. Rudys, L.E. Kavraki and D. S. Wallach, "Robotics-based location sensing using wireless Ethernet”, Wireless Networks, vol. 11, no. 1-2, (2005).

[6] E. Horvitz and J. Krumm, "LOCADIO: inferring motion and location from WiFi signal strengths", In: Proceedings of the 1st Annual International Conference on Mobile and Ubiquitous Systems: Networking and Services, Boston, USA, (2004) August 22-26.

[7] J. Hightower and G. Borriello, "Particle filters for location estimation in ubiquitous computing: a case study", In: Proceedings of the 6th International Conference on Ubiquitous Computing, Nottingham. (2004) September 07-10; Nottingham, England.

[8] T. Roos, P. P. Myllymaki, H. Tirri, P. Misikangas, J. Sievanen, “A probabilistic approach to WLAN user location estimation", International Journal of Wireless Information Networks, vol. 9, no. 3, (2002).

[9] K. Wu, J. Xiao and Y. Yi, "FILA: Fine-grained indoor location", The 31st Annual IEEE International Conference on Computer Communications, (2012) January 7-9; Orlando, USA.

[10] Y. Chen, Q. Yang, J. Yin and X. Chai, "Power efficient access-point selection for indoor location estimation”, IEEE Transactions on Knowledge and Data Engineering, vol. 18, no. 7, (2006).

[11] M. Sjoberg, M. Koskela, V. Viitaniemi, J. Laaksonen, "Indoor location recognition using fusion of SVM-based visual classifiers", IEEE International Workshop on Machine Learning for Signal Processing (MLSP 2010), Kittila, Finland, (2010) August 29. 
[12] S. Souvik, R. Bozidar, R. Roy Choudhury and T. Minka, "You are facing the Mona lisa: Spot localization using PHY layer information", Proceedings of the Mobisys'12, Lake District, UK, (2012) January 11-13.

[13] P. Bahl and V. N. Padmanabhan, "RADAR: an in-building RF-based user location and tracking system", In: Proceedings of the 19th Annual Joint Conference of the IEEE Computer and Communications Societies, (2000); Tel Aviv, Israel: IEEE.

[14] X. Luo, W. J. O'Brien and C. L. Julien, "Comparative evaluation of Received Signal-Strength Index (RSSI) based indoor localization techniques for construction jobsites. Advanced Engineering Informatics, vol. 25, no. 2, (2011).

[15] X. Wang, S. Yuan. R. Laur and W. Lang, "Dynamic localization based on spatial reasoning with RSSI in wireless sensor networks for transport logistics", Sensors and Actuators A-Physical, vol. 171, no. 2, (2011).

[16] Liu Zhi-Gao, Li Chun-wen, Geng Shao-Bo, Wu Dan-Chen and Ding Qing-Qing, "A personnel global positioning system in tunnel networks with blind areas", Journal of China Coal Society, vol. 35, no. 1, (2010).

[17] Han Dong-Sheng, Yang Wei, Liu Yang and Zhang Yu, "A weighted centroid localization algorithm based on received signal strength indicator for underground coal mine", Editorial Office of Journal of China Coal Society, vol. 38, no. 3, (2013).

\section{Authors}

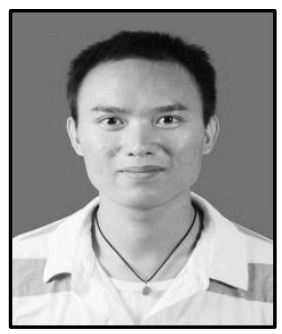

Yongxing Wang, $\mathrm{He}$ is currently working toward the PH.D. degree in China University of Mining and Technology, his research interests include Wireless Localization and Artificial Intelligence.

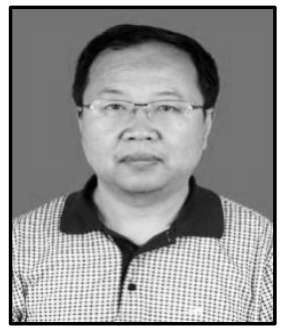

Gang Hua, He is currently a Professor in School of Information and Electrical Engineering with the China University of Mining and Technology. His current research interests include Artificial Intelligence, Safety Monitoring, Personal Localization and Image Processing. 